\title{
Condiciones psicométricas de la Escala de Acontecimientos Vitales Estresantes usada en población colombiana
}

\section{Psychometric Conditions of the Stressful Life Events Scale Used on Colombian Population}

\author{
Constanza Londoño Pérez ${ }^{1}$ \\ Marcela Velasco Salamanca ${ }^{2}$ \\ Carlos Pardo Adames ${ }^{3}$ \\ Marcela Patricia Escobar Martínez ${ }^{4}$ \\ Yeimy Yelitce Quintero Pulido ${ }^{5}$ \\ Luisa Fernanda Reyes Pareja ${ }^{6}$
}

\section{Universidad Católica de Colombia, Facultad de Psicología, Colombia}

\begin{abstract}
Resumen. Objetivo. Determinar las condiciones psicométricas desde el modelo TRI y evaluar la permanencia a través del tiempo de su calidad métrica determinada desde la Teoría Clásica de los Test de la Escala de Acontecimientos Vitales Estresantes. Muestra. Fue de 1225 colombianos entre 17 y 64 años $(M=33.14$; DT $=$ 12.47) de edad y ambos sexos, estratificada por sexos y procedencia. Metodología. Se analizaron las condiciones psicométricas de la escala desde la Teoría de Respuesta al Ítem (TRI), se mejoró el formato de presentación, se definieron límites de interpretación de los puntajes, y se analizó la diferencia en el impacto psicológico provocado por cada evento de acuerdo al sexo. Resultados. Los resultados muestran que la escala cuenta con un nivel adecuado de validez y confiabilidad, que el cambio de formato facilita su diligenciamiento y que es necesario ratificar si existen diferencias de sexo que deben ser estudiadas.
\end{abstract}

Palabras clave. Estrés, validez, confiabilidad, eventos vitales, impacto psicológico.

Abstract. Objective. This paper seeks to find the psychometric qualities of validity and reliability of the Scale of Stressful Life Events for Colombian people. Sample. 1225 participants aged between 17 and 64, $(M=33.14$ years old; $S D=12.47)$ stratified by gender and origin. Method. We analyzed the psychometric conditions of the scale from the Item Response Theory (IRT) improved the format of presentation, defined limits of interpretation of the scores, and analyzed the difference in psychological impact caused by each event according to sex. Results. The results show that the scale has an adequate level of validity and reliability, that the change of format facilitates its correct fulfillment, and that the need to study differences between the sexes must be assessed further.

Keywords. Stress, validity, reliability, vital stressful events.

${ }^{1}$ Constanza Londoño Pérez. Grupo de Investigación ENLACE, Facultad de Psicología, Universidad Católica de Colombia, Colombia. Coordinadora del Nodo Psicología y Salud de la Red de Investigadores de Ascofapsi. Vicepresidenta Región Andina Alapsa. E-mail: clondono@ucatolica.edu.co

${ }^{2}$ Marcela Velasco Salamanca. Universidad Católica de Colombia, Colombia. E-mail: rmvelasco@ucatolica.edu.co ${ }^{3}$ Carlos Pardo Adames. Universidad Católica de Colombia, Colombia. E-mail: capardo@ucatolica.edu.co

${ }^{4}$ Marcela Patricia Escobar Martínez. Universidad Católica de Colombia, Colombia. E-mail: mpescobar@ucatolica.edu.co ${ }^{5}$ Yeimy Yelitce Quintero Pulido. Universidad Católica de Colombia, Colombia. E-mail: yeyequintero@ucatolica.edu.co

${ }^{6}$ Luisa Fernanda Reyes Pareja. Universidad Católica de Colombia, Colombia. E-mail: 1freyes@ucatolica.edu.co

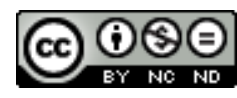

Esta obra está bajo una licencia de Creative Commons Reconocimiento-NoComercial-SinObraDerivada 4.0 Internacional. 


\section{Introducción}

El estrés agudo mantenido es un problema mundial de salud mental que ha sido relacionado con la aparición y gravedad de buena parte de las enfermedades crónicas, con la reducción de la productividad, el incremento del gasto en salud y por ende, con el mantenimiento de la pobreza, la inseguridad y la violencia (Alarcón, 2003; Banco Mundial, 2015; Lazarus \& Folkman, 1986; Organización Mundial de la Salud, 2014; Suárez. 2010).

El estrés aparece en relación con un grupo de acontecimientos universalmente capaces de provocarlo, en mayor o menor magnitud, en casi cualquier persona, independientemente de su género, procedencia o condición social (Barret \& Turner, 2005; Bodgan, Nikolova, \& Pizzagalli, 2013; Mazure, 1998; Oliva, Jiménez, Parra, \& Sánchez- Queija, 2008). Su impacto psicológico o dimensión de daño dependen de la reactividad individual (Acuña, Botto, \& Jiménez, 2009; Barcelata, Durán, \& Gómez-Maqueo, 2012; Castro et al., 2011; Ordaz \& Luna, 2012; Sandín, 2003; Solís \& Vidal, 2006). A este grupo de situaciones se les ha denominado Eventos Vitales Estresantes (AVE) (Folkman, 2001; Lazarus \& Folkman, 1986).

En 1967, Thomas Holmes y Richard Rahe determinaron la existencia de una lista de 43 eventos estresantes transculturales que podían causar enfermedad. Los autores dieron una puntuación asignada a cada uno de los acontecimientos y la suma de las respuestas es interpretada con una escala que indica que los puntajes menores a 150 indican 30\% de riesgo, es decir riesgo bajo, de presentar enfermedad psicosomática; entre 150 y 300 indica un 50\% de riesgo, es decir riesgo medio; y por encima de 300 un $80 \%$ de riesgo, es decir riesgo alto de presentar enfermedad psicosomática (Suárez, 2010). La escala es usada para evaluar la existencia de condiciones ambientales provocadoras de estrés y la intensidad de su efecto, fue adaptada al español por Oliva, Jiménez, Parra y Sánchez-Queijá (2008).

Los autores originales de la escala indican que algunos de los AVES afectan a quien los vive dependiendo de la edad, por ello se han propuesto listas específicas para adultos, adolescentes y niños; estas listas son presentadas como escalas que incluyen eventos económicos, escolares, laborales, familiares, del grupo de referencia, de la pareja y circunstancias estresantes de índole personal como eventos de salud o pérdidas materiales (González de Rivera, Revuelta, \& Morera Fumero 1983; Holmes \& Rahe, 1967).

Por otro lado, González de Rivera, Revuelta y Morera Fumero (1983) consideraron que puede haber diferencias culturales en la lista de AVE, además de la necesidad de validar la escala en español. Sus resultados mostraron que los españoles participantes de su estudio presentaban diferencias importantes con los norteamericanos en la valoración de algunos ítems y el impacto psicológico; situación que sin duda puede ser similar en la población colombiana aunque tenga cercanía en el idioma con España.

Ya han pasado más de cincuenta años desde el desarrollo de la Escala AVE basada en estudios en culturas pasadas. Se ha dado una gran transformación en los estilos de vida en el mundo y cambios generacionales importantes, representados en modificación de condiciones de vida, interacciones sociales, valoración individual de los eventos y transformación de normas, actitudes, hábitos y conductas en la población del mundo (Molarius et al., 2007). Todos estos cambios de las condiciones ambientales, políticas, 
económicas y sociales, que pueden provocar modificaciones en las condiciones estresantes, generan la necesidad de evaluar si la lista de AVE aún es la misma o es preciso ajustarla. Del mismo modo, se precisa determinar su posible uso en poblaciones como la colombiana; especialmente, porque esta escala ha tenido un uso extendido con fines diagnósticos e investigativos que, orientan importantes decisiones en salud sin que hayan sido evaluadas de nuevo las condiciones psicométricas actuales en población colombiana.

El paso del análisis de las condiciones psicométricas de la AVE mediante el uso de la Teoría Clásica de los Test a la Teoría de Respuesta al Ítem, permite optimizar la medición de constructos psicológicos. Ya que la unidad de análisis son los ítems, se facilita el uso de indicadores no dependientes de la toma aleatorizada de muestras difíciles de lograr (Muñiz, 1997), ofrece un análisis funcional entre el rasgo evaluado de quien responde y el ítem usado para evaluarlo (Luzardo \& Rodríguez, 2015). Se aportan datos específicos acerca del ajuste del modelo que sustenta al concepto base que, pretende evaluar el instrumento del instrumento y la relación entre la respuesta y el grado en que manifiesta el modelo (Abad, Olea, Ponsoda, \& García, 2011) el cual, para este caso, es el modelo de acontecimientos capaces de provocar la respuesta de estrés en la persona respondiente.

Por tanto, la presente investigación se planteó como objetivo determinar las condiciones psicométricas desde el modelo TRI y evaluar la permanencia a través del tiempo de su calidad métrica, determinada desde Teoría Clásica de los Test de la Escala de Eventos Vitales Estresantes en términos de validez, confiabilidad y condiciones de calificación e interpretación en población colombiana. Se pretende, también, indagar a nivel exploratorio la probable diferencia de sus condiciones de diseño y su uso en hombres y mujeres.

\section{Método}

El presente es un estudio psicométrico desde la Teoría de Respuesta al Ítem que permitió determinar, desde una nueva perspectiva, la posible utilidad actual de una prueba diseñada y validada hace más de 50 años. Lo anterior se hace considerando que, en este periodo de tiempo, pudo haber variado su nivel de validez y confiabilidad.

\section{Participantes}

Los participantes hacen parte de una muestra no aleatoria elegida por edad, sexo y procedencia, conformada por 1225 participantes hombres y mujeres colombianos entre los 17 y los 64 años de edad $(M=33.14$; $D T=12.47)$, con grupos de 245 personas provenientes de 5 ciudades colombianas. Se accedió a la población mediante el contacto en empresas, instituciones educativas y eventos sociales en los que se llevó a cabo la recolección de datos. Desde la TRI se permite analizar la relación entre la respuesta dada por la persona a cada ítem y el rasgo latente, se puede calcular la probabilidad de dar cada tipo de respuesta, cada nivel del estrés como rasgo medido, y no es requerida la elección aleatorizada de la población (Birnbaum, 1968; Rasch, 1960).

\section{Instrumentos}

Ficha de registro de información sociodemográfica. Es un formato de recolección de datos de identificación como sexo, edad, procedencia, nivel educativo, estado civil y estrato. 
Escala de Acontecimientos Vitales Estresantes. Desarrollada por Holmes y Rahé (1967), traducida al español por Oliva, Jiménez, Parra y Sánchez-Queijá (2008), consta de 23 ítems que presentan una lista de sucesos potencialmente estresantes en las que quien responde valora de 0 a 100 el impacto psicológico que le provoco el evento. Ambas versiones de escalas, la original en inglés y la traducida al español, tienen un $\alpha \geq .70$ que indica un nivel de consistencia interna adecuado. Tal como lo indican Oliva et al. (2008), para determinar la acumulación del impacto y el nivel de afectación de los acontecimientos estresantes vividos, se suman los acontecimientos estresantes reportados en el último año y se promedia el resultado del impacto psicológico con el que se ha calificado cada evento, para obtener el puntaje global de confiabilidad.

\section{Procedimiento}

Se define la cantidad de participantes de acuerdo con sexo, edad y procedencia, luego se contactó a la población con la autorización institucional para obtener el consentimiento informado a nivel personal, una vez se estableció la plena libertad de hacer parte de la investigación, se convocó a grupos de 30 personas en promedio y se les aplicó el instrumento. Después de que todos respondieran la escala completa, se les ofreció en grupo una breve orientación acerca de cómo manejar el estrés.

Se hizo un análisis estadístico descriptivo de las condiciones sociodemográficas de los participantes, y a nivel psicométrico, se realizó un análisis de la dimensionalidad de los resultados para el instrumento. Con base en el Análisis de Componentes Principales de los residuos del modelo de Rasch, se obtuvo un valor propio de 2.1, considerado dentro del límite para afirmar que, la prueba es unidimensional a pesar que los eventos estresantes valorados son muy diversos y que, inclusive, se pueden clasificar en diferentes tipos (Linacre, 2018).

\section{Resultados}

Los 1225 participantes eran 526 hombres (43\%) y 699 mujeres (57\%) colombianos entre los 17 y los 64 años de edad $(M=33.14 ; D T=12.47)$, provenientes de Bogotá, Medellín, Bucaramanga, Barranquilla y Cali.

Para mejorar la presentación de la escala, se ajustó el formulario de aplicación del cuestionario de tal modo que, las personas valoraran el impacto psicológico de cada evento en una casilla de respuesta específica. Los análisis de las propiedades psicométricas se realizaron bajo la Teoría de Respuesta al Ítem con el programa Winsteps ${ }^{\circledR}$ y se determinó que el cuestionario tiende a la exactitud. Ya que los errores de medida se acercaron a cero, puede considerarse confiable, pues el índice de separación es menor que 2 (tabla 1).

Se analizan los índices infit para identificar si existen respuestas anómalas y los oufit para identificar comportamientos anómalos ubicados al extremo opuesto del puntaje general por participante. Si uno de los indicadores cumple con al menos la combinación de infit por debajo de 1 y por encima de 1.3 o de un outfit por encima de 2 , denota funcionamiento ajustado de los ítems al modelo de Rash; no obstante, es importante revisar la redacción y el contenido de los ítems 4, 6, 10,11, 13 al 19 (tabla 2). 


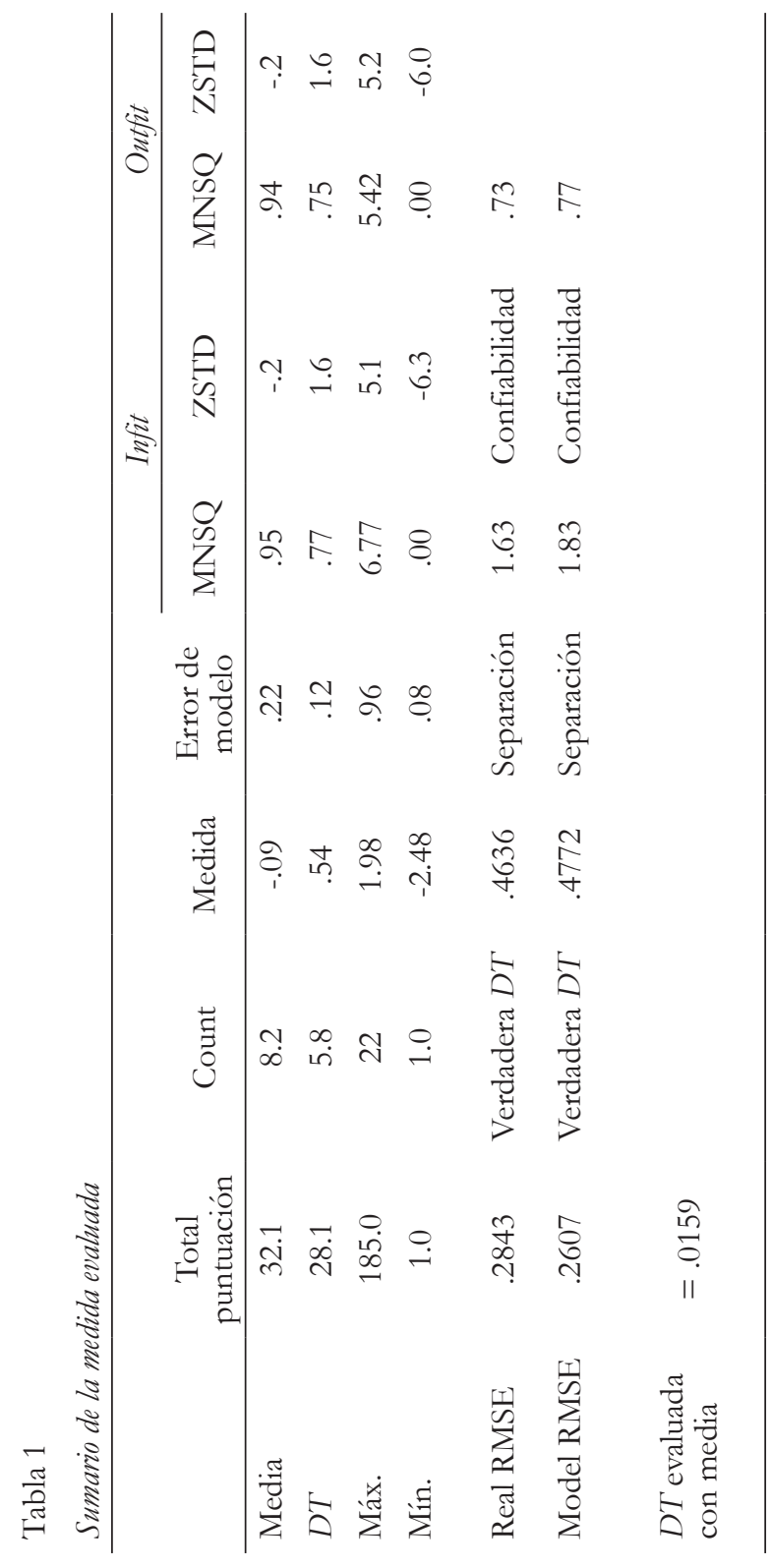

Adicional a los valores de ajuste para cada ítem, se obtuvieron los valores de la correlación punto biserial (tabla 2) que indican la discriminación. Los datos corresponden a valores altos de correlación lo que indica que hay una buena discriminación entre quienes responden sobre los eventos estresantes. Estos datos contribuyen con evidencias adicionales para la validez general de los resultados del instrumento, desde la perspectiva de aspectos analíticos proporcionados por lo resultados psicométricos (AERA, APA, \& NCME, 1999; Messick, 1995). 


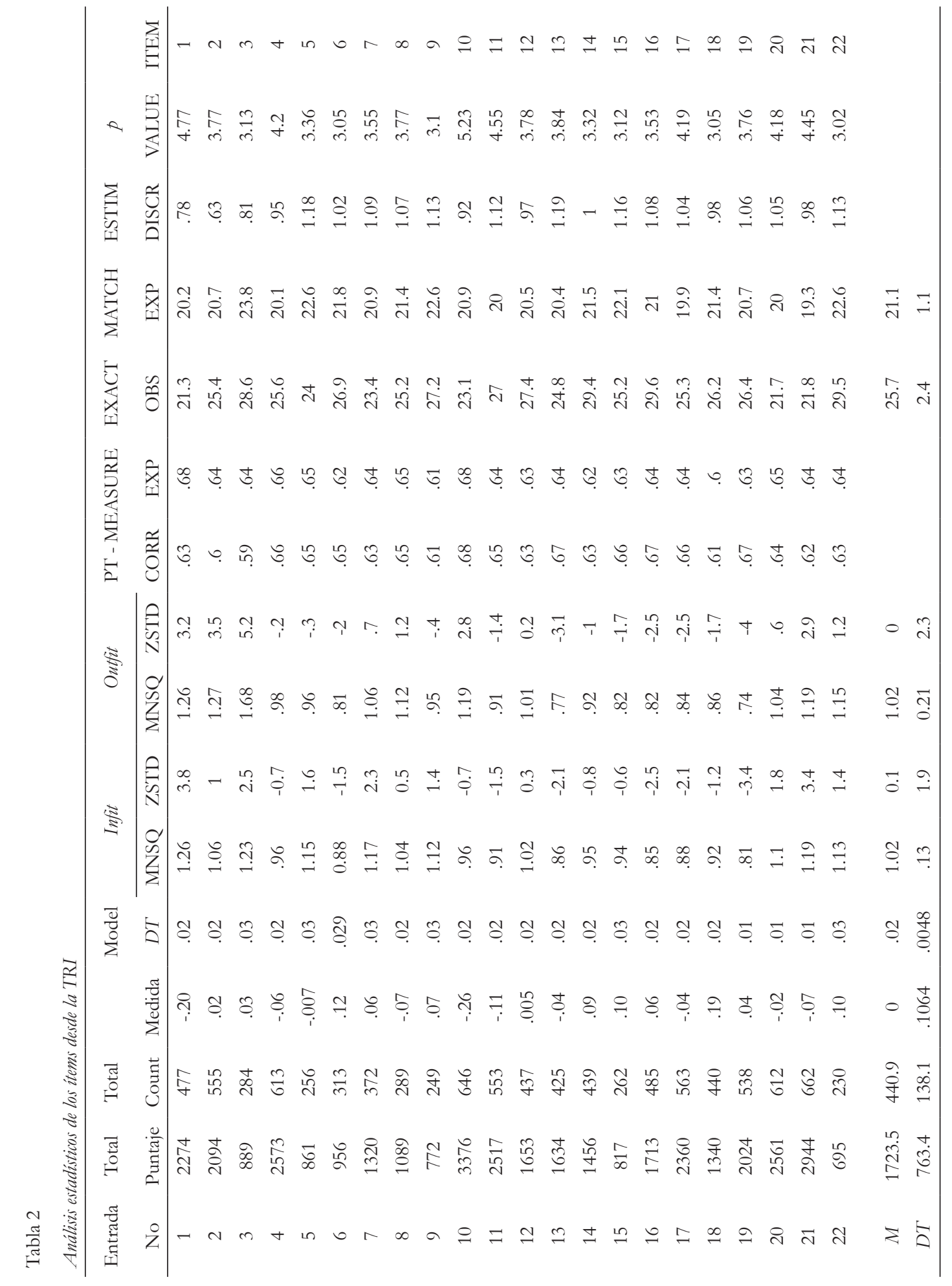

Actualidades en Psicologia, 33(126), enero-junio 2019, 83-96 


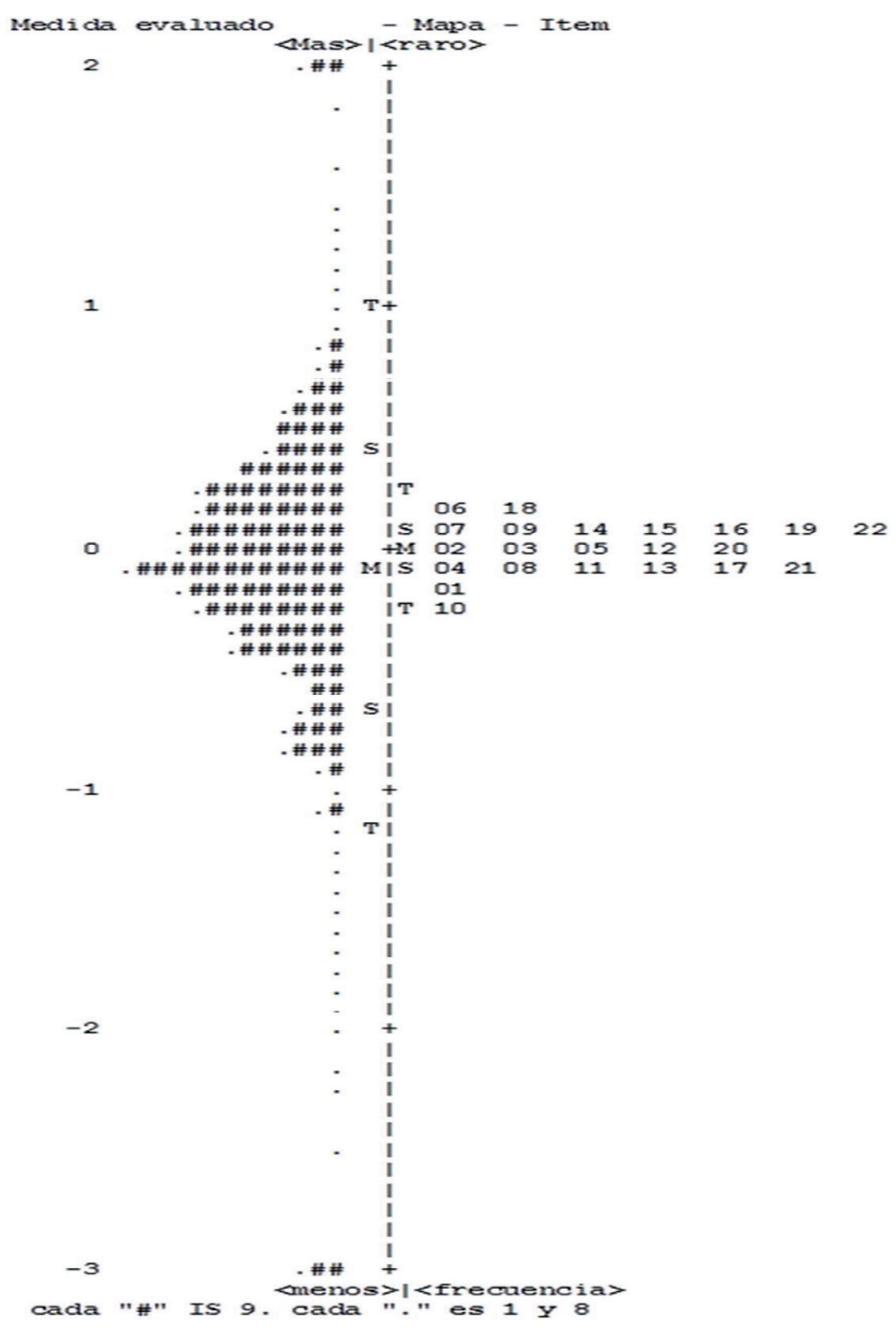

Figura 1. Tendencia de los puntajes brutos de los reactivos

La tendencia de los puntajes brutos de los reactivos sin subgrupos de ítems, se ubican de tal modo que, indican unidimensionalidad de constructo; además, se permite evidenciar que los ítems 4, 8, 11, 1, 17 y 21 son los más marcados y con mayor nivel de impacto referido por el grupo poblacional (Figura 1).

Los eventos vitales estresantes más reportados por los participantes fueron: vacaciones, problemas legales, despido del trabajo y cambio de hábitos alimentarios; en los hombres los más frecuentes fueron: logro personal notable, navidad, vacaciones, cambio de salud de un familiar y cambio en los hábitos de dormir. Entre los más reportados por las mujeres fueron lesión personal o enfermedad, navidades, cambio de salud de un familiar y logro personal notable y vacaciones. 
Por otra parte, los tres eventos con mayor puntaje promedio de impacto psicológico en la población general, fueron un logro personal notable $(M=62.3)$, la muerte de un familiar cercano $(M=57.7)$ y el cambio en las condiciones de vida $(M=55.5)$; para los hombres fueron el logro personal notable $(M=68.6)$, la navidad $(M=63.3)$ y el cambio en las condiciones de vida. Para las mujeres, los tres eventos fueron, la muerte de un familiar cercano $(M=57.6)$, el logro personal notable $(M=53.0)$ y la muerte de un amigo íntimo $(M=48.6)($ tabla 3$)$.

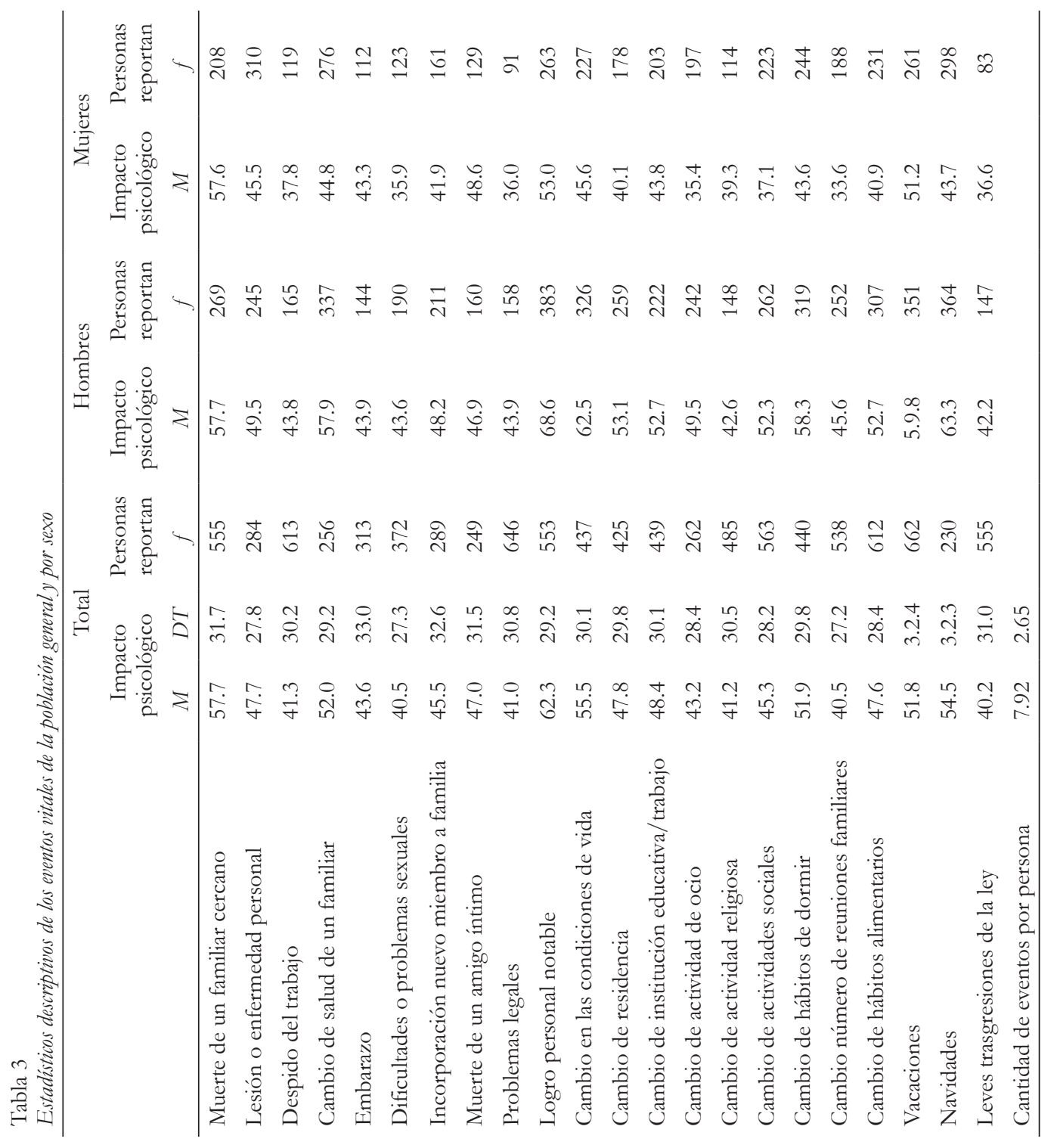


Como parte de los propósitos anexos a la validación de la prueba, en los que se pretende explorar la diferencia en los eventos vitales estresantes que afectan a hombres y mujeres, se compararon las diferencias de medias del impacto psicológico reportado por hombres y mujeres. Se encontró que existen diferencias significativas $(\phi \leq .05)$ en eventos como el cambio de salud de un familiar, dificultades o problemas sexuales, logro personal notable y en cambio de condiciones de vida, residencia, institución educativa/trabajo, actividad religiosa, social, hábitos de sueño, cantidad de reuniones familiares y en hábitos alimentarios; así como en vacaciones y leves trasgresiones de la ley; con un impacto psicológico en todos los casos significativamente más alto en los hombres y por ello es preciso hacer análisis futuros al respecto en nuevos estudios (tabla 4).

Tabla 4

ANOVA del impacto psicológico por sexo

\begin{tabular}{lcccc}
\hline & $\begin{array}{c}\text { Suma de } \\
\text { cuadrados }\end{array}$ & gl & $F$ & $p$ \\
\hline Muerte de un familiar cercano & .003 & 1 & .00 & 0.99 \\
Lesión o enfermedad personal & 22.41 & 1 & 2.92 & .087 \\
Despido del trabajo & 24.91 & 1 & 2.75 & .098 \\
Cambio de salud de un familiar & 259.62 & 1 & 31.93 & .000 \\
Embarazo & .22 & 1 & .02 & .888 \\
Dificultades o problemas sexuales & 44.56 & 1 & 6.06 & .014 \\
Incorporación nuevo miembro a la familia & 35.94 & 1 & 3.40 & .066 \\
Muerte de un amigo íntimo & 13.98 & 1 & .199 & .656 \\
Problemas legales & 35.28 & 1 & 3.76 & .054 \\
Logro personal notable & 382.35 & 1 & 45.63 & .000 \\
Cambio en las condiciones de vida & 178.70 & 1 & 21.05 & .000 \\
Cambio de residencia & 82.40 & 1 & 9.29 & .002 \\
Cambio de institución educativa/trabajo & 213.80 & 1 & 28.09 & .000 \\
Cambio de actividad de ocio & 7.17 & 1 & .77 & .380 \\
Cambio de actividad religiosa & 279.90 & 1 & 37.92 & .000 \\
Cambio de actividades sociales & 297.50 & 1 & 35.51 & .000 \\
Cambio de hábitos de dormir & 153.36 & 1 & 21.77 & .000 \\
Cambio número de reuniones familiares & 182.36 & 1 & 23.50 & .000 \\
Cambio de hábitos alimentarios & 517.00 & 1 & 53.68 & .000 \\
Vacaciones & 625.90 & 1 & 65.62 & .000 \\
Navidades & 16.75 & 1 & 1.74 & .188 \\
Leves trasgresiones de la ley & 518.01 & 1 & 62.24 & .000 \\
\hline & & & & \\
\hline
\end{tabular}




\section{Discusión y Conclusiones}

En conclusión, la Escala de Eventos Vitales Estresantes es de utilidad para evaluar el número de eventos vitales afrontados y el grado de impacto psicológico provocado en población entre 17 y 60 años tanto en hombres como en mujeres. Los resultados de confiabilidad y consistencia interna indican que la escala cuenta con un nivel de confiabilidad y validez adecuados y corresponde a un solo constructo o concepto "eventos vitales estresantes".

Esto revela que a pesar de los importantes cambios de las condiciones ambientales, económicas y sociales que enfrentan las personas en el mundo y en el país, dados en cerca de 50 años que lleva de creación de la AVE, la escala mantiene el nivel de calidad psicométrica y puede ser usada la versión actual. Por ello la escala es de utilidad en contextos clínicos de salud y en general, en condiciones en donde es requerido valorar el impacto psicológico y su efecto sobre el bienestar actualmente.

Las diferencias entre hombres y mujeres hacen necesario realizar nuevas investigaciones, a fin de determinar si es preciso desarrollar normas de calificación por sexo y construir baremos específicos de interpretación de los puntajes obtenidos por sexo. Sin duda, es de suma importancia notar que los hombres, en la totalidad de eventos vitales estresantes, reportaron haber vivido mayor impacto psicológico que las mujeres.

Dicha situación indica la probable existencia de una cierta vulnerabilidad a la afectación psicológica ante los eventos en este grupo que podría estar asociada a la baja sensación de controlabilidad de los eventos y del despliegue de recursos de afrontamiento aprobados socialmente, tal como se halló en estudios anteriores (Acuña, Botto, \& Jiménez, 2009; Álvarez \& Londoño, 2012; Castro et al., 2011; Londoño, Peñate, \& González, 2016; Ordaz \& Luna, 2012).

Respecto al estrés, la población analizada se encuentra expuesta a un nivel alto de estrés, ya que reporta más de cinco eventos vitales estresantes acumulados en el último año y, ello puede estar asociado al agotamiento de los recursos económicos y personales debido a la acumulación de impacto de los eventos que, se ve reflejada en la aparición de estrés crónico debida a la falta de tiempo necesaria para la recuperación psicológica una vez el evento ha ocurrido.

Asimismo, el estrés ha sido asociado a la aparición y agudización de problemas de salud física y mental, especialmente cuando es crónico, pues parece mermar y agotar los recursos de afrontamiento de los individuos (Almeida et al., 2011; Instituto Nacional de Salud Mental, 2013); en esta misma línea, Bodgan, Nikolova y Pizzagalli (2013) encontraron que la magnitud del estrés vivido está asociada con la gravedad, frecuencia y probabilidad de ocurrencia de eventos de depresión y ansiedad.

Aunque no se puede perder de vista que el estrés se produce no solo debido a las características del evento, sino que depende, en gran medida, de los recursos de afrontamiento de quien está expuesto a ellos, mediado por la evaluación que hace este acerca de la magnitud del evento y de la propia capacidad de respuesta (Lazarus \& Folkman, 1986) y que existen diferencias individuales que hacen que una persona sea más o menos reactiva al estrés (Folkman, 2001; Sandín, 2002; Solis \& Vidal, 2006). 
Vale la pena decir que las condiciones inestables de una región o de un país pueden ser facilitadoras de la existencia de estrés crónico, especialmente en la población masculina de una sociedad eminentemente machista que le impone no solo un papel protector y proveedor, sino que, además, los expone de forma directa a situaciones de riesgo derivadas de su papel en el manejo y resolución del conflicto interno en el caso de Colombia.

En suma, la escala de eventos vitales estresantes puede ser usada para valorar el grado de estrés al que está expuesta una persona y el grado de impacto psicológico percibido, sin perder de vista características personales como el sexo y la edad y el momento en el que es aplicada. También es posible reafirmar que la reactividad al estrés es altamente dependiente de las condiciones individuales y que el impacto psicológico es una medida subjetiva del grado de estrés provocado que debe ser considerada en el momento de estudiar el afrontamiento de los distintos eventos estresantes.

\section{Limitaciones y futuras direcciones}

Ya que en el presente estudio no se analiza el grado de cambio de la vida se espera que, en próximos estudios, se analice la posibilidad de incorporar a la escala de eventos vitales estresantes un componente dirigido a evaluarlo y determinar su impacto en la percepción del estrés en distintas poblaciones.

\section{Referencias}

Abad, F., Olea, J., Ponsoda, V., \& García, C. (2011). Medición en Ciencias Sociales y de la Salud. Madrid: Síntesis.

Abramson, L. Y., Alloy. L., Hankin, B., Haeffel, G., MacCoon, D., \& Gibb, B. (2002). Cognitive Vulnerability-Stress Model of Depression in a self-regulatory and psychobiological context. En I. Gotlib \& C. Hammen (2002). Handbook of Depression. (2 ed., pp. 268-294). New York: Guilford Press.

Abramson, L. Y., Seligman, M. E., \& Teasdale. J. D. (1978). Learned helplessness in humans: Critique and reformulation. Journal of Abnormal Psychology, 87(1), 49-74. doi: $10.1037 / 0021-843 X .87 .1 .49$

Acuña, L. (2012). Efectos de diversas variables sobre la salud. Acta de Investigación Psicológica, 2(3), 825-841. Recuperado de http://www.psicologia.unam.mx/ contenidoEstatico/archivo/files/Publicaciones/AIP

Acuña, J., Botto, B., \& Jiménez, J. (2009). Psiquiatría para atención primaria y el médico general. Depresión, ansiedad y somatización. Gaceta de Psiquiatría Universitaria, 5(4). 423-554. Recuperado de http://www.mediterraneo.cl

AERA, APA, \& NCME. (1999). Standards for educational andpsychological testing. Washington: AERA.

Alarcón, R. (2003). Mental health and mental health care in Latin America. World Psychiatry, 2(1), 54-56. PMCID: PMC1525063

Almeida, O., Alfonso, H., Pirkis, J., Kerse, N., Sim, M., Flicker, L., ... Pfaff, J. (2011). A practical approach to assess depression risk and to guide risk reduction 
strategies in later life. International Psychogeriatrics, 23(2). 280-291. doi: 10.1017/ S1041610210001870

Álvarez, N. \& Londoño, C. (2012). Diseño y validación del Cuestionario de Depresión en Hombres. (Tesis de Maestría). Universidad Católica de Colombia: Colombia.

Banco Mundial. (2015). El estrés, aliado de la pobreza de Latinoamérica. Recuperado de http:/ / www.bancomundial.org/es/news/feature/2015/12/28/el-estres-aliado-de-lapobreza-de-latinoamerica

Barcelata, B., Durán, E., \& Gómez-Maqueo, L. (2012). Valoración subjetiva de los sucesos de vida estresantes en dos grupos de adolescentes de zonas marginadas. Salud Mental, 35(6) 513-520. Recuperado de http://www.scielo.org.mx/scielo. php?pid $=$ S0185-3252012000600009yscript $=$ sci_ar ttext

Barrett, A. \& Turner, R. (2005). Family structure and mental health: The mediating effects of socioeconomic status family process and social stress. Journal of Health Social Behavior, 46(2), 156-169. doi: 10.1177/002214650504600203

Birnbaum, A. (1968). Some latent trait models and their use in inferring an examinee's ability. En F. Lord \& M. Novick (Eds.). Statistical Theories of Mental Test Scores. Reading, MA: Addison Wesley.

Bogdan, R., Nikolova, Y., \& Pizzagalli, D. (2013). Neurogenetics of depression: A focus on reward processing and stress sensitivity. Neurobiology of Disease, 52, 12-23. doi: 10.1016/j.nbd.2012.05.007

Castro, J., Diessler, J., Varea, E., Márquez, C., Larsen, M., Cordero, I., \& Sandi, C. (2011). Personality traits in rats predict vulnerability and resilience to developing stressinduced depression-like behaviors. HPA axis hyper-reactivity and brain changes in pERK1/2 activity. Psychoneuroendocrinology, 37(8). 1209-1223. doi: 10.1016/j. psyneuen.2011.12.014

Folkman, S. (2001). Revised coping theory and the process of bereavement. En M. S. Stroebe \& R. O. Hansson (Eds.). Handbook of bereavement research: Consequences. coping and care (pp. 563-584). Washington, DC: American Psychological Association.

Galea, S., Ahern,J., Nandi, E., Tracy, M., Beard,J, \& Vlavov. D. (2007). Urban neighborhood poverty and the incidence of depression in a population-based cohort study. Annals of Epidemiology, 17(3), 171-179. doi: 10.1016/j.annepidem.2006.07.008

Gómez, C., \& Londoño, C. (2013). Validación preliminar de la escala de eventos vitales estresantes para jóvenes. Documento de trabajo. Universidad Católica de Colombia: Colombia.

González de Rivera, J. L., Revuelta, J. L. \& Morera Fumero. A. (1983). La valoración de sucesos vitales: Adaptación española de la escala de Holmes y Rahe. Psiquis, 4(1), 7-11. Recuperado de http://www.psicoter.es/_arts/83_A032_02.pdf

Halkin, R. (2012). A Generalization of the Dirichlet. Distribution, 33(11), 1-18. doi: $10.18637 /$ jss.v033.i11 
Hammen, C. (2005). Stress and depression. Annual Review of Clinical Psychology, 1, 293-319.

Holmes, T. \& Rahe, R. (1967). The social readjustment rating scale. Journal of Psychosomathic Response, 11, 213-218. doi: 10.1016/0022-3999(70)90007-3

Instituto Nacional de Salud Mental. (2013). Depresión. Recuperado de http://www.nimh. nih.gov/health/publications/espanol/depresion/depresion.pdf

Latkin, C. \& Curry, A. (2003). Stressful neighborhoods and depression: A prospective study of the impact of neighborhood disorder. Journal of Health and Social Behavior, 44(1), 34-44. doi: 10.2307/1519814

Lazarus, R. S. (1993). Coping theory and research: past, present, and future. Psychosomatic Medecine, 55, 234-247. doi: 10.1016/0272-7358(86)90029-2

Lazarus, R. S. \& Folkman, S. (1986). Estrés y Procesos Cognitivos. Madrid, España: Martínez Roca.

Linacre, J. M. (2018). Winsteps ${ }^{\circledR}($ Version 4.3.1) [Computer Software]. Beaverton, Oregon: Winsteps.com. Retrieved from https://www.winsteps.com/

Londoño, C., Peñate, W., \& González, M. (2016). Validación Diferencial y Discriminante del Cuestionario de Depresión para Hombres (CDH). Terapia Psicológica, 16(34), 129-142. doi: 10.4067/S0718-48082016000200005

Luzardo, M. \& Rodríguez, P. (2015). A Nonparametric Estimator of a Monotone Item Characteristic Curve. In L. A. van der Ark, D. Bolt, W.Ch. Wang, J. A. Douglas and S. M. Chow (Eds), Quantitative Psychology Research (pp. 99-108). Suiza: Springer International Publishing.

Mazure, C. M. (1998). Life stressors as risk factors in depression. Clinical Psychology Sciences and Practical, 5, 291-313. doi: 10.1111/j.1468-2850.1998.tb00151.x

Messick, S. (1995). Validity of Psychological Assessment: Validation of Inferences From Persons' Responses and Performances as Scientific Inquiry Into Score Meaning. American Psychologist, 50(9), 741-749. Retrieved from https://www.d.umn. edu/ kgilbert/ened5560-1/Validity\%20-\%20Messick1995.pdf

Molarius, A., Berglund, K., Eriksson, C., Lambe, M., Nordström, E., Eriksson, H. G., \& Feldman, I. (2007). Socioeconomic conditions, lifestyle factors, and self-rated health among men and women in Sweden. European Journal of Public Health, 17(2), 125-133. doi: 10.1093/eurpub/ck1070

Monroe, S. M. \& Hadjiyannakis, K. (2002). The social environment and depression: Focusing on severe life stress. In I. H. Gotlib \& C. L. Hammen. Handbook of depression (pp. 314-340). New York: Guilford Press

Muñiz, J. (1997). Introducción a la Teoría de Respuesta a los Ítems. Madrid: Pirámide.

Oitzl, M., Champagne, D., Van Der Veen, R., \& de Kloet. W. (2010). Brain development under stress: hypotheses of glucocorticoid actions revisited. Neuroscience Biobehavior Review, 34(6), 853-85. doi: 10.1046/j.1460-9568.2000.00231.x 
Oliva, A., Jiménez, J., Parra, A., \& Sánchez-Queija, I. (2008). Acontecimientos vitales estresantes, resiliencia y ajuste adolescente. Revista de Psicopatología y Psicología Clínica, 13(1), 53-62. doi: 10.5944/rppc.vol.13.num.1.2008.4050

Ordaz, S. \& Luna, B. (2012). Sex differences in physiological reactivity to acute psychosocial stress in adolescence. Psychoneuroendocrinology, 37(8), 1135-1157. doi: 10.1016/j.psyneuen.2012.01.002

Organización Mundial de la Salud. (2014). Informe sobre la situación mundial de las enfermedades no transmisibles 2014. Recuperado de http://apps.who.int/iris/bitstream/ handle/10665/149296/WHO_NMH_NVI_15.1_spa.pdf;jsessionid=4BE16F B636F325AD865B0B3D04BE4895? sequence $=1$

Pizzagalli, D. A., Jahn, A., \& O 'Shea, J. P. (2005). Toward an objective characterization of an anhedonic phenotype: a signal-detection approach. Biological Psychiatry, 57(4-15), 319-327. doi: 10.1016/j.biopsych.2004.11.026

Rasch, G. (1960). Probabilistic Models for Some Intelligence and Attainment Tests. Copenhague: The Danish Institute for Educational Research.

Sandín, B. (2003). El estrés: Un análisis basado en el papel de los factores sociales. Revista Internacional de Psicología Clínica y de la Salud, 3(1), 141-157. Recuperado de http:/ / www.aepc.es/ijchp/articulos_pdf/ijchp-65.pdf

Safford, M. M., Russell, L. B., Suh, D. C., Roman, S., \& Pogach, L. (2005). How much time do patients with diabetes spend on self-care? Journal American of Board Familiars Practice, 18, 262-70. doi: 10.3122/jabfm.18.4.262

Solís, C. \& Vidal, A. (2006). Estilos y estrategias de afrontamiento en adolescentes. Revista de Psiquiatríay Salud Mental Hermilio Valdiza, 6, 33-39. Recuperado de http://www. hhv.gob.pe/revista/2006/3\%20ESTILOS\%20Y\%20ESTRATEGIAS\%20 DE\%20AFRONTAMIENTO.pdf

Suárez, M. (2010). La importancia del análisis de los acontecimientos vitales estresantes en la práctica clínica. Revista Médica La Pa₹, 16(2), 58-62. Recuperado de http: / / www.scielo.org.bo/scielo.php?script=sci_arttext\&pid=S172689582010000200010\&lng=es\&tlng=es 